\title{
Severe asthma: adding new evidence - Latin American Thoracic Society
}

\author{
Gabriel García ${ }^{1}$, Miguel Bergna ${ }^{2}$, Juan C. Vásquez $\mathbb{1}^{3}$, Maria C. Cano Salas ${ }^{3}$, \\ José L. Miguel ${ }^{3}$, Carlos Celis Preciado ${ }^{4}$, Agustin Acuña Izcaray $\mathbb{1}^{5}$, \\ Manuel Barros Monge ${ }^{6}$, Natalia García Batista ${ }^{7}$, Ignacio Zabert ${ }^{8}$, \\ José L. Mayorga ${ }^{9}$, Renato Casanova Mendoza ${ }^{10}$, Martha Gutierrez ${ }^{11}$, \\ Maria F. Montero Arias ${ }^{12}$, Luzcenelia Urtecho Perez ${ }^{13}$, Miguel Antúnez ${ }^{14}$, \\ Victoria Williams Derby ${ }^{15}$, Alejandra Villatoro Azméquita ${ }^{16}$, Luis Motiño ${ }^{17}$, \\ M. Alenita De Oliveira ${ }^{18}$, Diana Rey Sanchez ${ }^{19}$, Marisol Arroyo ${ }^{9}$ and \\ Mario Rodriguez ${ }^{9}$
}

Affiliations: ${ }^{1}$ Asthma Dept, Latin American Thoracic Society, Montevideo, Uruguay. ${ }^{2}$ Respiratory Unit, Hospital Centrángolo, Vicente López, Argentina. ${ }^{3}$ Respiratory Unit, Instituto Nacional de Enfermedades Respiratorias, México, DF, Mexico. ${ }^{4}$ Respiratory Unit, Hospital Universitario San Ignacio, Bogotá, Colombia. ${ }^{5}$ Pneumology Dept, Hospital Universitario de Caracas, Caracas, Venezuela. ${ }^{6}$ Escuela de Medicina, Universidad de Valparaíso, Hospital Van Buren, Valparaiso, Chile. ${ }^{7}$ Sociedad Dominicana de Neumología y Cirugía de Tórax, Santiago, Dominican Republic. ${ }^{8}$ Asociación Argentina de Medicina Respiratoria, Neuquén, Argentina. ${ }^{9}$ A2DAHT Iberoamerican Agency for Development and Assessment of Health Technology, Mexico, DF, Mexico. ${ }^{10}$ Sociedad Peruana de Neumología, Lima, Perú. ${ }^{11}$ Sociedad Uruguaya de Neumología, Montevideo, Uruguay. ${ }^{12}$ Asociación Costarricense de Neumología y Cirugía de Tórax, San José, Costa Rica. ${ }^{13}$ Asociación Nicaragüense de Neumología, Nicaragua, Nicaragua. ${ }^{14}$ Sociedad Chilena de Enfermedades Respiratorias, Santiago, Chile. ${ }^{15}$ Asociación Panameña de Neumología y Cirugía de Tórax, Panamá, Panama. ${ }^{16}$ Asociación Guatemalteca de Neumología y Cirugía de Tórax, Guatemala, Guatemala. ${ }^{17}$ Asociación Hondureña de Neumología y Cirugía de Tórax, Honduras, Honduras. ${ }^{18}$ Sociedad Brasilera de Neumología y Tisiología, São Paulo, Brasil. ${ }^{19}$ Asociación Colombiana de Neumología y Cirugía de Tórax, Bogotá, Colombia.

Correspondence: Gabriel García, Asociación Latinoamericana de Tórax - Asthma, Mario Cassinoni 1689 Montevideo, Uruguay. E-mail: gabrielgarcia.cepirágmail.com

ABSTRACT This document constitutes a summary of the clinical practice guidelines (CPGs) prepared at the initiative of the Latin American Thoracic Society (ALAT). Due to new evidence in the treatment of severe asthma, it was agreed to select six clinical questions, and the corresponding recommendations are provided herein. After considering the quality of the evidence, the balance between desirable and undesirable impacts and the feasibility and acceptance of procedures, the following recommendations were established. 1) We do not recommend the use of an inhaled corticosteroid (ICS) plus formoterol as rescue medication in the treatment of severe asthma. 2) We suggest performing many more high-quality randomised studies to evaluate the efficacy and safety of tiotropium in patients with severe asthma. 3) Omalizumab is recommended in patients with severe uncontrolled allergic asthma with serum IgE levels above 30 IU. 4) Anti-interleukin (IL)-5 drugs are recommended in patients with severe uncontrolled eosinophilic asthma (cut-off values above 150 cells $\mu \mathrm{L}^{-1}$ for mepolizumab and above 400 cells $\mu \mathrm{L}^{-1}$ for reslizumab). 5) Benralizumab is recommended in adult patients with severe uncontrolled eosinophilic asthma (cut-off values above 300 cells $\mu \mathrm{L}^{-1}$ ). 6) Dupilumab is recommended in adult patients with severe uncontrolled allergic and eosinophilic asthma and in adult patients with severe corticosteroid-dependent asthma.

@ERSpublications

Severe \#asthma: evidence @ALATorax https://bit.ly/34jYhg2

Cite this article as: García G, Bergna M, Vásquez JC, et al. Severe asthma: adding new evidence Latin American Thoracic Society. ERJ Open Res 2021; 7: 00318-2020 [https://doi.org/10.1183/ 23120541.00318-2020].

Received: 12 June 2020 | Accepted after revision: 21 Sept 2020

Copyright $\odot$ ERS 2021. This article is open access and distributed under the terms of the Creative Commons Attribution Non-Commercial Licence 4.0. 


\section{Introduction}

Asthma is a global health problem, with $\sim 300$ million people affected. It is estimated that $\sim 5 \%$ of the population suffers from asthma, although some reports indicate that this proportion may be higher in certain age groups. This represents a medical challenge and at the same time a significant health burden for both patients and health institutions. The morbidity and mortality from asthma is increasing [1-3]. The prevalence of asthma in Latin America has been reported with averages of 17.3\% (6-7 years old) and 15.8\% (13-14 years old) [4]. However, epidemiological studies in adults are limited (Mexico 5\%, Colombia 6.3\%) [5]. Previous studies have revealed that most asthma patients in Latin America are not being treated, many suffer frequent exacerbations and there is almost no awareness of the severity of the disease, not even among patients treated in specialised centres where other individuals also suffer from severe asthma $[6,7]$. Furthermore, Latin American physicians have not shown much adherence to the recommendations of the clinical practice guidelines (CPGs) [8]. This regional situation and the recognition of the importance of serious types of asthma motivated the Latin American Thoracic Society (ALAT) Asthma Department to bring together a group of experts from all over the region to develop a CPG on the diagnosis and management of severe asthma. Given recent evidence and new analysis methods, such as indirect comparisons for biological drugs in the treatment of severe asthma, our group has selected six questions from the CPG, which are presented herein.

\section{Method}

\section{Guideline Development Group}

The Guideline Development Group (GDG) is composed of pulmonologists specialised in asthma, members of the ALAT Asthma Department and methodological experts with expertise in the development of systematic literature reviews (SRs) and CPGs (figure 1). A core group met on multiple occasions through a network platform and had two face-to-face meetings. Scope of the document and clinical questions were agreed upon, and in the second meeting, the content and wording of the recommendations endorsed in the modified Delphi panel were reviewed in detail. Panel members disclosed all possible conflicts of interest. Those with relevant conflicts of interest participated in the discussions about evidence but did not participate in the formulation of recommendations.

\section{Structured clinical questions}

The GDG designed the whole clinical questionnaire. It was sought to be clear, precise and specific to facilitate the search and review of scientific evidence and thus avoid recommendations that are not well adjusted to the clinical problems posed by the CPG. The PICO (Population, Intervention, Comparasion, Outcome) model was considered in most of the questions.

\section{Comprehensive search for scientific evidence}

The evidence was identified following internationally validated algorithms and strategies. MeSH terms (Medical Subject Headings) were identified and used to build a sensitive and specific search strategy [9]. A preliminary search of relevant CPGs was carried out, followed by the identification, evaluation and synthesis of all the relevant evidence.

In the comprehensive search for SRs, different search strategies were developed, and several databases were used. The Cochrane Library, the Campbell Collaboration Library of Systematic Reviews, the Centre for Reviews and Dissemination databases (includes Database of Abstracts of Reviews of Effects (DARE)), the National Institute for Health Research (UK) (NIHR), the Database of Promoting Health Effectiveness

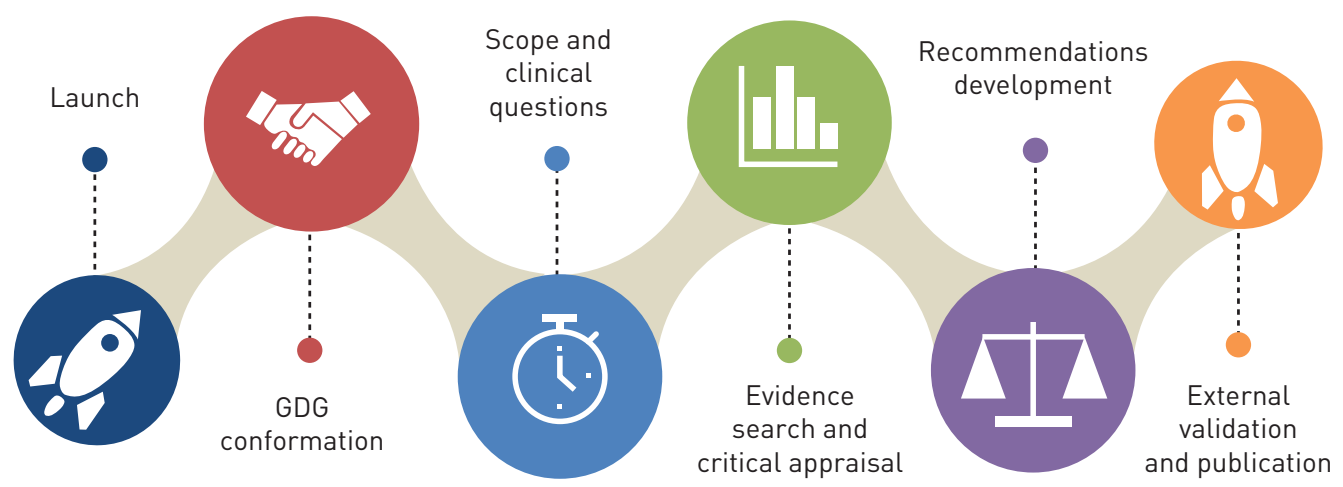

FIGURE 1 Overall development process of clinical practice guidelines. GDG: Guideline Development Group. 
Reviews (DoPHER), The ripDatabase, Medline and PubMed (the National Library of Medicine in the USA), and Embase, and the National Institute for Health and Care Excellence (NICE) were consulted to identify high-quality health technology assessments (HTAs). The databases that were consulted to identify published clinical studies were the following: the Cochrane Library up to 2017, the Cochrane Central Register of Controlled Trials, the Cochrane Database of Systematic Reviews, DARE (Issue 1 2017), Medline 1950-2019 (OVID), Embase 1980-2019 (OVID), CINAHL 1982-2019 (NLH Search 2.0), LILACS (1998 to 2019), ARTEMISA (1999 to 2019) and SciELO (1999 to 2019).

\section{Quality assessment and the ranking of evidence}

To establish the quality of the scientific evidence, the AGREE II tool [10] was used in the case of CPGs, while the AMSTAR II tool was used for SRs $[11,12]$. To assess the quality of the evidence, we chose to use the scale developed by the Scottish Intercollegiate Guidelines Network (SIGN) [13, 14], which uses two attributes to assess the quality of the scientific evidence (level of evidence): the study design and the risk of bias.

\section{Expert Formal Consensus}

A Delphi panel was carried out as a process to collect the opinion of experts representing the respiratory societies associated with ALAT $[15,16]$. Expert Formal Consensus (EFC) members received an invitation via e-mail to review each of the clinical recommendations placed on a digital platform designed for this purpose and assigned a rating using a 'Likert scale'. A mean level of 7.0 was established as the minimum level of consensus, and $70 \%$ of responses were in the range of 7-9 on the Likert scale. GN members interacted among the participants, processing the information and filtering the relevant content. They modified the recommendations according to the clinical arguments of all panellists to send the new text to a new round of Delphi panel.

\section{Drafting of the recommendations}

GN members met on several occasions to review all the evidence related to the structured clinical questions and, according to the level of evidence, to be able to determine the grade of recommendation and the wording. The recommendations also considered the risk versus benefit ratio to guide decision-making. In accordance with SIGN, a careful analysis of evidence was carried out, as well as an analysis of the experience of the GDG members and the accessibility to diagnostic tests and therapeutic interventions, to qualify clinical recommendations and to express the level of confidence the GDG had in the recommendations.

\section{Results}

To correctly prepare the body of the guideline, 17 clinical questions were selected (table 1). A total of 157 SRs were found in PubMed and 21 SRs in Embase with the search strategies used. Specific search strategies were carried out for some of the therapeutic interventions to complement the information, and 167 additional SRs were found. In some cases, such as antileukotrienes and theophylline, search strategies were conducted to identify randomised controlled trials (RCTs), and 301 abstracts were located. Three rounds of the modified Delphi panel were performed. Of the 17 clinical questions analysed, only one, question 3 , referring to the definition of difficult-to-treat asthma, did not reach a minimum level of consensus, a mean of 7.0 and a percentage of $70 \%$ in the Delphi panel; therefore, it was rejected, as agreed upon by the members of the GDG (table 2). Thus, 16 questions formed part of the clinical recommendations of the guideline. The final recommendations are outlined in table 3 and are detailed below. An analysis of six questions and their recommendations are presented in this document by consensus.

\section{Clinical recommendations}

Question 7: What is the indication for the on-demand use of the combined therapy of an ICS and formoterol associated with maintenance treatment with an ICS and an LABA in patients with severe asthma?

Justification

The use of a combination of an inhaled corticosteroid (ICS) plus a long-acting bronchodilator (LABA) through a single inhaler or single maintenance and relief therapy (SMART) has been suggested to treat mild to moderate asthma. The question that arises is whether such a strategy can be indicated in patients with severe asthma.

\section{Research}

Exhaustive research was carried out (157 documents in PubMed, 21 in Embase, 497 in the TRIP database and four in different databases), and four documents were selected (one clinical practice guide, three SRs). 


\section{TABLE 1 Latin American Thoracic Society (ALAT) Severe Asthma Guidelines questions}

1) What is the definition of severe asthma?

2) What criteria are used to diagnose uncontrolled asthma?

3) What criteria are used to diagnose difficult-to-treat asthma?

4) What are the risk factors identified and associated with the development of severe asthma?

5) What are the phenotypes of severe asthma?

6) What are the predictive biomarkers for therapy response of patients with severe asthma?

7) What is the indication for the on-demand use of the combined therapy of an ICS and formoterol associated with maintenance treatment with an ICS and a LABA in patients with severe asthma?

8) Is there any additional benefit in adding theophylline in patients with severe asthma?

9) Is there any additional benefit in adding antileukotrienes in patients with severe asthma?

10) Is there any additional benefit when adding a long-acting anticholinergic drug (tiotropium) to ICS and LABA treatment in patients with severe asthma?

11) What is the efficacy and safety of anti-IgE monoclonal antibodies in the treatment of severe asthma in children and adults?

12) What is the efficacy and safety of anti-IL-5 monoclonal antibodies in the treatment of severe asthma in children and adults?

13) What is the efficacy and safety of anti-IL-5 receptor monoclonal antibodies in the treatment of severe asthma in children and adults?

14) What is the efficacy and safety of methotrexate in the treatment of severe asthma in children and adults?

15) What is the efficacy and safety of anti-IL- 4 and IL-13 monoclonal antibodies in the treatment of severe asthma in children and adults?

16) What is the efficacy and safety of macrolides in the treatment of severe asthma in children and adults?

17) What is the efficacy and safety of bronchial thermoplasty in the treatment of severe asthma in children and adults?

ICS: inhaled corticosteroid; LABA: long-acting bronchodilator; IL: interleukin.

\section{Evidence summary}

An SR published by CATES and LASSERSON [15] included three clinical trials, one of which was performed with symptomatic patients with asthma exacerbation in the year prior to the study but no diagnosis of severe asthma. A significant reduction in the number of hospitalisations (OR 0.68, 95\% CI: $0.40-1.16$ ) and severe exacerbations was reported when the combination of an ICS and formoterol versus terbutaline was used (OR 0.54, 95\% CI: 0.44-0.65). Another SR published by the same authors [16] reported that patients with mild to moderate asthma, non-severe according to the 2014 American Thoracic Society (ATS)/ European Respiratory Society (ERS) criteria, treated with a combination of budesonide and formoterol as rescue and maintenance therapy instead of the use of an ICS registered a lower use of rescue medicine (OR $-0.16,95 \%$ CI: -0.27 to -0.05 ). Regarding the safety of using formoterol versus terbutaline, the first had less of an effect on serum potassium, heart rate, blood pressure and the QT interval. The 2014 ATS/ ERS guidelines suggested that reducing the LABA dose improves the control of asthma in children with asthma of any degree of severity; therefore, there is no specific recommendation for the use of $\beta_{2}$-agonist bronchodilators [3].

Recently, SOBIERAj et al. [17] published an SR meta-analysis that included 16 controlled clinical trials (22748 patients). The authors found that patients with persistent asthma treated with the SMART strategy had a decreased risk of asthma exacerbations. In this meta-analysis, the authors included three clinical trials conducted in patients who received high doses of an ICS. Unfortunately, none of the three studies met the 2014 ATS/ERS criteria for treating severe asthma. The evidence found for patients aged 4 to 11 years was limited.

\section{Conclusions and recommendations}

We have not found high-quality evidence that shows efficacy and safety for the addition of a combination of an ICS plus formoterol as rescue treatment to standard therapy in patients with severe asthma. Considering extrapolated data from different populations, the use of a combination of an ICS plus formoterol as rescue treatment in patients who have the same maintenance combination is suggested to improve symptoms quickly (level of evidence 1+, grade of recommendation B). Further high-quality randomised studies are needed to evaluate the efficacy and safety of using a combination of an ICS plus formoterol as a rescue treatment in patients with severe asthma (conditional recommendation). 


\section{TABLE 2 Statistics of the modified Delphi panel}

\begin{tabular}{|c|c|c|c|c|c|c|}
\hline \multirow{2}{*}{$\begin{array}{l}\text { Question number } \\
\text { ON - } 1\end{array}$} & \multicolumn{2}{|c|}{$\begin{array}{l}\text { Round } 1 \text { mean } \\
\text { agreement \% }\end{array}$} & \multicolumn{2}{|c|}{$\begin{array}{l}\text { Round } 2 \text { mean } \\
\text { agreement \% }\end{array}$} & \multicolumn{2}{|c|}{$\begin{array}{l}\text { Round } 3 \text { mean } \\
\text { agreement \% }\end{array}$} \\
\hline & 7.0 & 67 & 7.4 & 89 & 8.5 & 100 \\
\hline \multicolumn{7}{|l|}{ FN - 1} \\
\hline ON - 2 & 7.0 & 67 & 7.2 & 78 & & \\
\hline \multicolumn{7}{|l|}{ FN - 2} \\
\hline ON - 3 & 6.2 & 56 & 6.4 & 72 & $E$ & $E$ \\
\hline \multicolumn{7}{|l|}{$F N-E$} \\
\hline ON - 4 & 7.4 & 83 & & & & \\
\hline \multicolumn{7}{|l|}{ FN - 3} \\
\hline ON - 5 & 6.6 & 78 & 7.6 & 83 & & \\
\hline \multicolumn{7}{|l|}{ FN -4} \\
\hline ON - 6 & 7.5 & 83 & & & & \\
\hline \multicolumn{7}{|l|}{ FN - 5} \\
\hline ON - 7 & 7.5 & 78 & & & & \\
\hline \multicolumn{7}{|l|}{ FN - 6} \\
\hline ON - 8 & 8.3 & 89 & & & & \\
\hline \multicolumn{7}{|l|}{ FN - 7} \\
\hline ON - 9 & 7.4 & 83 & & & & \\
\hline \multicolumn{7}{|l|}{ FN - 8} \\
\hline ON - 10 & 6.1 & 56 & 7.6 & 94 & 8.3 & 100 \\
\hline \multicolumn{7}{|l|}{ FN - 9} \\
\hline ON - 11 & 7.3 & 78 & & & & \\
\hline \multicolumn{7}{|l|}{ FN - 10} \\
\hline ON - 12 & 7.9 & 89 & & & & \\
\hline \multicolumn{7}{|l|}{ FN - 11} \\
\hline ON - 13 & 8.1 & 89 & & & & \\
\hline \multicolumn{7}{|l|}{ FN - 12} \\
\hline $\mathrm{ON}-14$ & 8.7 & 100 & & & & \\
\hline \multicolumn{7}{|l|}{ FN - 13} \\
\hline ON - 15 & 7.4 & 78 & & & & \\
\hline \multicolumn{7}{|l|}{ FN - 14} \\
\hline$O N-16$ & 7.0 & 67 & 7.6 & 89 & & \\
\hline \multicolumn{7}{|l|}{ FN - 15} \\
\hline ON - 17 & 7.3 & 78 & & & & \\
\hline$F N-16$ & & & & & & \\
\hline
\end{tabular}

The mean and agreement percentage of the modified Delphi panel were calculated. Questions 1 and 10 underwent major changes after the second round; therefore, the Guideline Development Group (GDG) decided to conduct a third round on both questions. ON: original number of the question; FN: final number of the question, E: eliminated question as agreed upon by the GDG.

Question 10: Is there any additional benefit when adding a long-acting anticholinergic drug (tiotropium) to ICS and LABA treatment in patients with severe asthma?

Justification

Long-acting anticholinergics (LAMAs) are indicated as a third option for the treatment of patients with moderate asthma; however, their benefit in patients with severe asthma is unknown, which is why the above question arises.

\section{Research}

Exhaustive research was carried out (157 documents in PubMed, 21 in Embase, 497 in the TRIP database and four in different databases), and four documents were selected (three SRs and one controlled clinical study).

\section{Evidence summary}

\section{Tiotropium}

An SR published by Rodrigo and Castro-Rodríguez et al. [18] included 13 clinical trials, 4966 patients, but only one trial was performed in patients classified as severe and with 48 weeks of treatment. Notably, in this study, the number of exacerbations suffered by patients in the previous year was unclear. 
TABLE 3 Recommendation summaries and the level of evidence

\begin{tabular}{|c|c|c|c|c|}
\hline & Recommendations & $\begin{array}{l}\text { Level of } \\
\text { evidence }\end{array}$ & $\begin{array}{l}\text { Grade of } \\
\text { recommendation }\end{array}$ & Judgement \\
\hline 6 & $\begin{array}{l}\text { There is not enough good-quality } \\
\text { evidence to support the on-demand use } \\
\text { of an ICS plus formoterol in patients } \\
\text { with severe asthma }\end{array}$ & $1+$ & $\mathrm{B}$ & Conditional \\
\hline 9 & $\begin{array}{l}\text { Tiotropium (LAMA) should be } \\
\text { considered as a third controller added } \\
\text { to ICS plus LABA treatment in children } \\
>6 \text { years of age and adults }\end{array}$ & $1-$ & B & Conditional \\
\hline 10 & $\begin{array}{l}\text { We recommend the use of omalizumab } \\
\text { in adult patients and children with } \\
\text { severe uncontrolled allergic asthma } \\
\text { (cut-off values }>30 \text { IU) }\end{array}$ & $\begin{array}{c}1+\text { ladults) } \\
1- \\
\text { (children) }\end{array}$ & $\begin{array}{l}A \\
A\end{array}$ & $\begin{array}{l}\text { Strong } \\
\text { Strong }\end{array}$ \\
\hline 11 & $\begin{array}{l}\text { We recommend the use of monoclonal } \\
\text { anti-IL-5 antibodies in patients with } \\
\text { severe uncontrolled eosinophilic } \\
\text { asthma (cut-off values }>150 \text { cells } \mu \mathrm{L}^{-1} \\
\text { for mepolizumab and }>400 \text { cells } \mu \mathrm{L}^{-1} \\
\quad \text { for reslizumab) } \\
\text { There is no evidence to recommend the } \\
\text { use of monoclonal anti-IL-5 antibodies } \\
\text { in children }\end{array}$ & $\begin{array}{c}1+\text { ladults) } \\
1- \\
\text { (children) }\end{array}$ & $\begin{array}{l}\text { A } \\
B\end{array}$ & $\begin{array}{l}\text { Strong } \\
\text { Conditional } \\
\text { recommendation for } \\
\text { use restricted to trials }\end{array}$ \\
\hline 12 & $\begin{array}{l}\text { We recommend the use of } \\
\text { benralizumab in patients with severe } \\
\text { uncontrolled eosinophilic asthma } \\
\text { (cut-off values }>300 \text { cells } \mu \mathrm{L}^{-1} \text { ) } \\
\text { There is no evidence to recommend the } \\
\text { use of monoclonal anti-IL-5 antibodies } \\
\text { in children }\end{array}$ & $\begin{array}{c}1+\text { ladults) } \\
1- \\
\text { (children) }\end{array}$ & $A$ & $\begin{array}{l}\text { Strong } \\
\text { Conditional } \\
\text { recommendation for } \\
\text { use restricted to trials }\end{array}$ \\
\hline 14 & $\begin{array}{l}\text { We recommend the use of dupilumab in } \\
\text { adult patients with severe allergic and } \\
\text { eosinophilic uncontrolled asthma and in } \\
\text { adult patients with severe } \\
\text { corticosteroid-dependent asthma } \\
\text { Lebrikizumab and tralokinumab have } \\
\text { not been able to demonstrate } \\
\text { consistent efficacy in the most } \\
\text { important outcomes in patients with } \\
\text { severe asthma; therefore, we do not } \\
\text { suggest their use }\end{array}$ & $\begin{array}{l}1+\text { ladults) } \\
1+\text { ladults) }\end{array}$ & $\begin{array}{l}A \\
A\end{array}$ & $\begin{array}{c}\text { Strong } \\
\text { Strong } \\
\text { recommendation } \\
\text { against }\end{array}$ \\
\hline
\end{tabular}

However, the SR results showed a decrease in the frequency of exacerbations (18.2\% versus $24.0 \%$ ), with an NNT (number needed to treat) of 17 and improvement in the results of forced expiratory flow (FEF) and forced expiratory volume in $1 \mathrm{~s}\left(\mathrm{FEV}_{1}\right)$, supporting the use of triple therapy. The quality of life (QoL) and symptoms also showed statistically significant improvement.

KEW and DAHRI [19] published an SR that included four clinical trials, 1197 asthma patients treated with a combination of an ICS and an LABA, where the diagnosis of severe asthma was at the investigator's discretion. The authors did not show data regarding previous exacerbations suffered by patients to establish if they had severe uncontrolled asthma. The results showed that those patients who received tiotropium as an adjuvant drug had fewer exacerbations requiring oral corticosteroids than those patients who received placebo. However, the difference was not statistically significant (OR 0.76, 95\% CI: 0.57-1.02). The authors reported a moderate level of evidence. The QoL evaluated by the Asthma Quality of Life Questionnaire (AQLQ) did not show significant differences (mean difference (MD) 0.09, 95\% CI: 0.03-0.20) and failed to decrease the incidence of adverse events (OR 0.60, 95\% CI: $-0.24-1.47$; $\mathrm{I}^{2}=76 \%$ ). 
Another SR published by RODRIGO AND CASTRO-Rodríguez [20] was carried out to evaluate the efficacy and safety of tiotropium in adolescents with moderate to severe asthma. The authors included three controlled clinical studies; however, two were conducted in patients with moderate asthma, and only one was carried out in patients with severe asthma for 12 weeks. The meta-analysis included the three studies, but no severity stratification was carried out to determine whether there were differences in patient groups. Taking into account the results of the only study that considered patients with severe asthma, the improvements in the pulmonary function tests, $\mathrm{FEV}_{1}(\mathrm{MD}-0.10,95 \% \mathrm{CI}:-0.21-0.01$ ), symptoms (ACQ-7) (RR 0.53, 95\% CI: $-0.15-1.78$ ) and incidence of exacerbations (RR 0.69, 95\% CI: -0.43-1.12) did not show statistically significant differences.

A study carried out in children from 6 to 11 years of age published by SzEFLER et al. [21] included symptomatic patients with severe asthma treated with a high-dose ICS plus an LABA or antileukotrienes or with a medium-dose ICS plus two additional controllers for 4 months prior to admission. For 12 weeks, patients received a dose of either $5 \mathrm{mg}$ or $2.5 \mathrm{mg}$ of tiotropium or placebo. When compared with placebo treatment, the results showed an improvement in $\mathrm{FEV}_{1}$ with the $5 \mathrm{mg}$ dose (adjusted mean difference (AMD) $139 \mathrm{~mL}, 95 \% \mathrm{CI}: 75-203$; $\mathrm{p}<0.001)$, but the improvement was not significant with the $2.5 \mathrm{mg}$ dose (AMD $139 \mathrm{~mL}, 95 \% \mathrm{CI}: 28-99 ; \mathrm{p}<0.27$ ). There were no statistically significant differences in the symptom

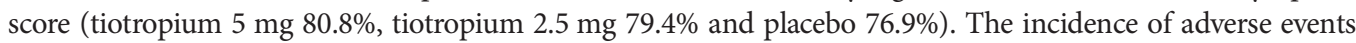
was lower in patients treated with tiotropium $5 \mathrm{mg}(\mathrm{n}=56 ; 43.1 \%)$ and tiotropium $2.5 \mathrm{mg}(\mathrm{n}=59 ; 43.4 \%)$ than in patients treated with placebo $(n=66 ; 49.3 \%)$. Most of the adverse events were mild to moderate.

\section{Conclusions and recommendation}

We suggest the use of tiotropium as a third controller due to its risk-benefit profile in patients with severe asthma. The use of tiotropium as an add-on to ICS/LABA treatment in children 6 years of age and adults with asthma shows a slight improvement in symptoms, pulmonary function and exacerbations. However, this is a weak recommendation due to the limited information available in clinical studies carried out in patients with severe asthma (level of evidence 1-, grade of recommendation B). Further high-quality randomised studies are needed to evaluate the efficacy and safety of using tiotropium in patients with severe asthma (conditional recommendation).

\section{Question 11: What is the efficacy and safety of anti-IgE monoclonal antibodies in the treatment of severe asthma in children and adults? \\ Justification}

Omalizumab is a humanised monoclonal antibody with high affinity for serum IgE, and it has been approved for the treatment of allergic asthma. There is new information regarding this drug; therefore, we have decided to reformulate this question.

\section{Research}

Exhaustive research was carried out (224 documents in PubMed, 21 in Embase, 497 in the TRIP database and four in different databases), and six documents were selected (three CPGs and three SRs).

\section{Evidence summary}

\section{Omalizumab}

An SR carried out by Normansell et al. [22] included 25 controlled clinical studies, seven of which were conducted in patients considered to have severe asthma. The meta-analyses of patients with severe asthma showed an improvement in the number of exacerbations in both patients treated with high doses of an ICS (OR 1.00, 95\% CI: 0.5-1.99; 277 patients) and those treated with an ICS plus oral corticosteroids (OR 1.65, 95\% CI: $0.66-4.13$; 95 patients). When compared to placebo, there was a significant improvement in asthma control (OR 1.69, 95\% CI: $1.26-2.26)$ and the QoL (57.5\% versus $38.6 \% ; \mathrm{p}<0.01$ ) and a reduction in the use of rescue medications (MD $-0.30,95 \% \mathrm{CI}: 0.49-0.10)$. As the results showed heterogeneity in the subgroup of patients with severe asthma, it was not possible to conduct a meta-analysis when analysing pulmonary function. Regarding the safety variables, there were no significant differences between the group that received omalizumab and the control group.

An HTA published by Norman et al. [23] selected 11 controlled clinical trials, one of which included paediatric patients. When compared to placebo, omalizumab reduced exacerbations in both adult patients (rate ratio (RT) 0.74; 95\% CI: 0.55-1.00) and paediatric patients (RT 0.66; 95\% CI: $0.44-1.00$ ). There is no solid evidence in children.

The ERS/ATS 2014 CPG [3] reported that, when comparing placebo with omalizumab, the latter improved the QoL (65\% versus 57\%; RR: 1.19, 95\% CI: 1.08-1.30; four studies) and asthma control (mean difference in ACQ score: $0.87,95 \%$ CI: 0.6-1.14; one study) and showed a reduction in the need for systemic 
corticosteroids (RR: 0.73 , 95\% CI: 0.56-0.94). In paediatric patients treated with omalizumab, the therapy reduced the need for corticosteroid dosage (MD: 14\%, 95\% CI: 5-21\%) and hospitalisation (MD: 5\%, 95\% CI: 1-6\%). However, omalizumab had no significant effect on the QoL or asthma control.

The ATS CPG 2018 [24], which based its recommendation mainly on a Cochrane SR published in 2014 [22], suggests the use of omalizumab treatment in patients older than 6 years with asthma inadequately controlled with high-dose inhaled corticosteroids and the use of at least one additional controller and who are sensitised to at least one aero allergen and present high IgE levels $\left(30-1300 \mathrm{IU} \cdot \mathrm{mL}^{-1}\right.$ in patients aged 6 to 11 years and $30-700 \mathrm{IU} \cdot \mathrm{mL}^{-1}$ in patients older than 12 years).

The GINA CPG 2019 recommendations [25] suggest the use of omalizumab in patients older than 6 years with moderate to severe asthma inadequately controlled in Step 4 of treatment and high levels of IgE.

\section{Indirect comparisons}

A "network meta-analysis" published by NACHEF et al. [26] compared omalizumab versus mepolizumab in patients 12 years of age and older. The authors included 18 studies with omalizumab (4854 patients) and four studies with mepolizumab (1620 patients). The results showed that there was no difference in the improvement in $\mathrm{FEV}_{1}$ with either drug; however, both were superior to placebo (omalizumab 138.05, 95\% CI: $83.08-193.01$ versus mepolizumab 147.32, 95\% CI: 116.36-178.28). When comparing both cases in relation to asthma control, no clinically significant difference was obtained in ACQ scores (mepolizumab $0.78,95 \%$ CI: $0.93-0.62$ versus omalizumab $0.76,95 \%$ CI: $1.15-0.37$ ) or in AQLQ scores (mepolizumab 0.82, 95\% CI: 0.71-0.92 versus omalizumab 1.2, 95\% CI: 1.11-1.28).

\section{Conclusions and recommendations}

We recommend the use of omalizumab in patients with severe uncontrolled allergic asthma and with serum IgE levels above $30 \mathrm{IU}$. We suggest the use of subcutaneous omalizumab in patients older than 6 years with severe asthma that is inadequately controlled. The benefit seems to outweigh the risks of presenting adverse events (level of evidence 1+, grade of recommendation A - adults) (level of evidence $1-$, grade of recommendation $\mathrm{A}$ - paediatric patients) (strong recommendation).

\section{Question 12. What is the efficacy and safety of anti-IL-5 monoclonal antibodies in the treatment of severe asthma in children and adults? \\ Justification}

IL-5 is a pro-eosinophilic type 2 cytokine that binds to its receptor, IL-5R, on eosinophils and basophils; it promotes the recruitment of eosinophils and their activation and contributes to eosinophilic inflammation of the airway. We currently have two humanised monoclonal antibodies against interleukin 5 (anti-IL5): mepolizumab and reslizumab. The answer to this question should be focused on reducing exacerbations and improving the QoL, pulmonary function and asthma control.

\section{Research}

Exhaustive research was carried out (218 documents in PubMed, 21 in Embase, 497 in the TRIP database and four in different databases), and nine documents were selected (nine systematic reviews).

\section{Evidence summary}

\section{Mepolizumab}

An SR and meta-analysis published by LiU et al. [27], which included three clinical studies of severe eosinophilic asthma, showed that mepolizumab therapy reduced the risk of exacerbations (OR 0.30, 95\% CI: $0.13-0.67, \mathrm{p}=0.004$ ) and significantly improved AQLQ scores (MD 0.26, 95\% CI: 0.03-0.49, p=0.03) compared with placebo. It was observed that mepolizumab significantly lowered eosinophil counts in sputum (MD 26.05\%, 95\% CI: 29.34-22.77\%, p=0.0003) and blood (MD 0.05 L, 95\% CI: 20.04-0.13 L, $\mathrm{p}=0.29)$.

YANCEY et al. [28] compared mepolizumab versus placebo and included four clinical trials $(\mathrm{n}=1388)$ of patients with severe eosinophilic asthma. Mepolizumab produced a $51 \%$ reduction in the rate of exacerbations requiring hospitalisation ( $R R$ 0.49; $95 \%$ CI $0.30-0.80 ; p=0.004$ ) and in emergency room visits (RR, 0.49; 95\% CI, 0.33-0.73; $\mathrm{p}<0.001)$ compared with placebo.

An SR published by Powell et al. [29] compared intravenous (i.v.) mepolizumab to placebo and reported a significant reduction in the exacerbation rate with the use of i.v. mepolizumab (RR $0.52,95 \% \mathrm{CI}$ : $0.43-0.64)$.

A post hoc meta-analysis of the SR published by FARNE et al. [30], performed on data from phase III, MENSA and MUSCA studies, showed a reduction in the mean exacerbation rate of $49-70 \%$ and an 
improvement in St. George's Respiratory Questionnaire (SGRQ) and ACQ-5 scores in patients treated with mepolizumab compared with patients treated with placebo.

\section{Reslizumab}

A meta-analysis comparing the effect of reslizumab versus placebo published by Li et al. [31] showed fewer exacerbations $(\mathrm{OR}=0.46,95 \% \mathrm{CI}: \quad 0.35,0.59, \mathrm{p}<0.00001)$ and lowered blood eosinophil counts (standardised mean difference (SMD) $-475.62,95 \%$ CI: -528.41 to $-422.83, \mathrm{p}<0.00001$ ), as well as improvements in $\mathrm{FEV}_{1}(\mathrm{SMD} 0.16,95 \% \mathrm{CI}: 0.10-0.23, \mathrm{p}<0.00001)$ and symptom control as determined by ACQ score (SMD -0.26 , IC $95 \%$ : -0.36 to $-0.16, \mathrm{p}<0.00001$ ), in patients treated with reslizumab.

\section{Indirect comparisons}

To assess the efficacy of mepolizumab and reslizumab, an SR and meta-analysis published by HeNRIKSEN et al. [32] showed a 53\% reduction in exacerbations (95\% CI: 46-59) in favour of both anti-IL-5 drugs compared to placebo. When compared with placebo, mepolizumab and reslizumab showed significant improvements in lung function (112.93 mL; 95\% CI: 82.44-143.31), asthma control ( -0.29 points; $95 \%$ CI: -036 to -0.23$)$ and asthma-related QoL (0.32; 95\% CI: 0.22-0.43).

An SR published by FARNE et al. [30] compared the effect of anti-IL5 and anti-IL-5R biological agents versus placebo. This review showed a reduction of exacerbations by using s.c. mepolizumab (RR $0.45,95 \%$ CI: 0.36-0.55), i.v. mepolizumab (RR 0.53, 95\% CI: 0.44-0.64), reslizumab (RR 0.43, 95\% CI: 0.33-0.55) and benralizumab (RR 0.62, 95\% CI: 0.55-0.70). Although an improvement in the QoL was observed, it did not exceed the minimal clinical difference for the ACQ or the SGRQ. An improvement in lung function was determined with all biological agents versus placebo. However, the improvement was statistically significant with s.c. mepolizumab (MD 0.11 L, 95\% CI: 0.06-0.17), i.v. mepolizumab (MD $0.08 \mathrm{~L}, 95 \% \mathrm{CI}: 0.02-0.15$ ), reslizumab (MD 0.11 L, 95\% CI: 0.07-0.15) and benralizumab (MD $0.10 \mathrm{~L}$, 95\% CI: 0.05-0.14). No serious adverse events were shown with any anti-IL5 compared with placebo.

NACHEF et al. [26] performed an efficacy comparison between mepolizumab and omalizumab. The authors found that there were no significant differences in the asthma control questionnaire score, $\mathrm{FEV}_{1}$ or peak expiratory flow rate. Both drugs reduced the calculated exacerbation rate per year by $50 \%$.

An SR published by CASALE et al. [33] indirectly compared reslizumab with benralizumab using a Bayesian network meta-analysis. Eleven studies were carried out in patients with severe eosinophilic asthma. Reslizumab significantly improved the ACQ score $(-0.37$; credible interval (CrI), -0.63 to $-0.10 ; \operatorname{Pr}=100 \%)$ and the AQLQ score (-0.32; CrI, 0.03 to 0.60; $\mathrm{Pr}=99 \%)$ compared with benralizumab. This indirect comparison suggested that reslizumab might be more effective than benralizumab in patients with eosinophilic asthma (benralizumab, $\geqslant 300$ cells $\mu \mathrm{L}^{-1}$; reslizumab, $\geqslant 400$ cells $\mu \mathrm{L}^{-1}$ ) and with two or more exacerbations in the previous year.

A network meta-analysis carried out by $\mathrm{HE}$ et al. [34], which evaluated the effect of anti-IL-5 and anti-IL-5R antibodies, showed significant improvements in FEV $_{1}$ (SMD 0.18; 95\% CI: 0.12-0.23; p<0.001) and in AQLQ scores (SMD 0.20; 95\% CI: 0.13-0.26; $\mathrm{p}<0.001$ ) in patients treated with monoclonal antibodies compared with those treated with placebo. There were no significant differences in exacerbation risks between individuals treated with monoclonal antibodies and those treated with placebo (RR 0.68 , 95\% CI: 0.11-4.14, $\mathrm{p}=0.097)$.

\section{Conclusions and recommendations}

Anti-IL-5 as an add-on treatment for patients with severe uncontrolled eosinophilic asthma is recommended. We suggest cut-off values of blood eosinophils above 150 cells $\mu \mathrm{L}^{-1}$ for the use of mepolizumab and above 400 cells $\mu \mathrm{L}^{-1}$ for the use of reslizumab. There is not enough evidence confirming the use of these drugs in children under 12 years of age (level of evidence 1+, grade of recommendation $\mathrm{A}-$ adults) (strong recommendation) (level of evidence 1-, grade of recommendation B - paediatric patients). (It is recommended to carry out research, and the use of these drugs is dependent on the results of clinical studies.)

Question 13. What is the efficacy and safety of anti-IL-5 receptor monoclonal antibodies in the treatment of severe asthma in children and adults?

Justification

IL-5 is a pro-eosinophilic Type 2 cytokine that binds to its IL-5R receptor expressed on eosinophils and basophils, promoting eosinophil recruitment and activation and contributing to eosinophilic inflammation of the airway. It is possible that patients with severe asthma have high blood and/or sputum eosinophil counts. Benralizumab is a monoclonal antibody that blocks the $\alpha$-chain of the IL-5 receptor. Thus, there is 
a need to answer this question in terms of reducing exacerbations and improving the QoL, lung function and asthma control.

Research

Exhaustive research was carried out (218 documents in PubMed, 21 in Embase, 497 in the TRIP database and four in different databases), and 10 documents were selected (10 SRs).

\section{Evidence summary}

Benralizumab

An SR, published by NICE in 2019 [35], included three clinical studies, and benralizumab reduced the annual exacerbation frequency by $43 \%$ compared to placebo (RR $0.57,95 \%$ CI: $0.47-0.69, \mathrm{p}<0.0001$ ). The results demonstrated the greatest benefit in patients with high blood eosinophils $\left(>300\right.$ cells $\left.\mu \mathrm{L}^{-1}\right)$ and in those patients who had more exacerbations over the 12 -month period prior to the study (four or more exacerbations requiring corticosteroid therapy).

An SR published by TIAN et al. [36], which included nine controlled clinical studies on 2321 patients, showed that exacerbation frequency increased $(38.66 \%)$ in patients treated with placebo compared to those receiving benralizumab therapy $(26.28 \%)$, who showed a significantly decreased risk of presenting exacerbations (RR 0.63, 95\% CI: 0.52-0.76, p<0.00001). Regarding pulmonary function tests, three studies did not find significant $\mathrm{FEV}_{1}$ differences in either group in comparison to the baseline values (SMD -0.10 , 95\% CI: $-0.31-0.10, \mathrm{p}=0.33$ ). The results of the meta-analysis regarding symptom control (ACQ score) showed a significant difference in favour of benralizumab versus placebo (SMD $-0.10,95 \% \mathrm{CI}$ : $-0.26-0.06$, $\mathrm{p}=0.22$ ) in patients with eosinophilic asthma, while regarding the QoL (AQLQ score), there were no differences (SMD $-0.11,95 \%$ CI: $-0.32-0.10, \mathrm{p}=0.3$ ). Regarding the incidence of adverse events, there were no differences between the benralizumab group (1216 of 1646) and the placebo group (622 of 847) (RR 1.00, 95\% CI: $0.95-1.05, \mathrm{p}=0.96$ ).

An SR carried out by LiU et al. in 2018 [37], which included the same studies (five CCTs) as other SRs, demonstrated that, compared with the placebo, benralizumab treatment helped in reducing exacerbations and improving lung function $\left(\mathrm{FEV}_{1}\right)$, the QoL and the control of disease (ACQ score). The most effective dose was $30 \mathrm{mg}$.

Recently, LIU et al. [38] published another SR of eight clinical studies. A meta-analysis showed that the group treated with benralizumab had a lower risk of experiencing general adverse events (RR 0.94, 95\% CI: $0.90-0.98$ ), serious adverse events (RR $0.82,95 \%$ CI: $0.68-0.98$ ) or asthma exacerbation (RR $0.72,95 \%$ CI: 0.61-0.85) than the group treated with placebo. The authors concluded that benralizumab showed an adequate and safe profile in the treatment of eosinophilic asthma.

\section{Indirect comparisons}

An SR published by CABON et al. [39] aimed to compare the clinical efficacy and safety of benralizumab with other anti-IL-5 monoclonal antibodies in patients with severe asthma. The authors included 10 clinical trials and 3421 patients. The network meta-analysis outcomes regarding the reduction in the exacerbation rate were best with reslizumab $3 \mathrm{mg}$ (51\%), followed by mepolizumab $750 \mathrm{mg}(22 \%)$ and mepolizumab $100 \mathrm{mg}$ (13\%). Regarding the Asthma Control Test, benralizumab was the most effective ( $\mathrm{MD}-0.38,95 \% \mathrm{CI}:-0.97$ to $-0.18, \mathrm{p}<0.01$ ). Reslizumab treatment was the most effective in improving the $\mathrm{FEV}_{1}$ values (MD 0.14 L, 95\% CI: 0.05-0.24, p<0.01). Regarding safety outcomes, benralizumab showed the best safety profile of all (RR $0.94,95 \%$ CI: $0.57-1.54$ ), but considering the incidence of serious adverse events, reslizumab had the best safety profile (RR $0.81,95 \%$ CI: $0.22-3.03$ ).

BOURDIN et al. [40] performed a comparison of benralizumab versus anti-IL-5 drugs $(\mathrm{n}=1524)$. After matching adjustment, benralizumab and mepolizumab reduced exacerbations compared with placebo by $52 \%$ and $49 \%$, respectively (RR $0.94,95 \%$ CI: $0.78-1.13$ ), and reduced the rate of exacerbations requiring hospitalisation by $52 \%$ and $52 \%$, respectively (RR 1.00, 95\% CI: 0.57-1.7524). An improvement in lung function was observed with the use of benralizumab $\left(0.10 \mathrm{~L}\right.$ in pre-bronchodilator $\left.\mathrm{FEV}_{1}\right)$ versus mepolizumab $\left(0.07 \mathrm{~L}\right.$ in pre-bronchodilator $\left.\mathrm{FEV}_{1}\right)(\mathrm{MD} 0.03 \mathrm{~L} ; 95 \% \mathrm{CI}$ : -0.06-0.12). It was difficult to generate a sufficiently effective sample size to produce a reliable estimate when comparing the benralizumab and reslizumab heterogeneous populations.

Another SR published by $\mathrm{HE}$ et al. [34] included 21 clinical studies that evaluated treatment with benralizumab, mepolizumab and reslizumab. In the "meta-analysis by pairs" extracted from 16 studies, an improvement in $\mathrm{FEV}_{1}$ was observed with the use of anti-IL-5 therapies versus placebo (SMD 0.18, 95\% CI: $0.12-0.23 ; \mathrm{p}<0.001)$. Regarding lung function, the network meta-analysis showed the efficacy of mepolizumab, reslizumab and benralizumab (NMA: SMD 1.09; 95\% CI: 1.04-1.15; 1.11; 95\% CI: 
$1.05-1.18 ; 1.10 ; 95 \%$ CI: $1.05-1.15$, respectively). The surface under the cumulative ranking curve showed the following results: reslizumab (SUCRA 77.7\% probability), benralizumab (SUCRA 63.4\% probability) and mepolizumab (SUCRA 58.9\% probability). Regarding AQLQ score improvement, reslizumab was the most effective (85.6\% probability), followed by benralizumab $(62.3 \%$ probability) and mepolizumab $(51.5 \%$ probability). There were no significant differences in the risk of exacerbation, but the SUCRA classification found that the risk of exacerbation was lowest with mepolizumab (23.1\% probability), followed by benralizumab (38.5\% probability) and finally reslizumab (57.6\% probability). Regarding safety, reslizumab showed the lowest risk of presenting adverse events (NMA: RR, 1.44, 95\% CI: 1.01-2.05).

As previously mentioned, CASALE et al. [33], when stating an indirect comparison, suggested that reslizumab might be more effective than benralizumab in patients with eosinophilic asthma (benralizumab, $\geqslant 300$ cells $\mu \mathrm{L}^{-1}$; reslizumab, $\geqslant 400$ cells $\mu \mathrm{L}^{-1}$ ) with two or more exacerbations in the previous year.

In another SR with network meta-analysis published by EDRIs et al. [41], the authors found no significant difference among those biological drugs. Anti-IL-5 drugs are the treatment alternatives with the largest number of clinical studies, and in regard to reducing exacerbations in eosinophilic asthma patients, they show superiority over placebo. However, the network meta-analysis outcomes did not show statistically significant differences among these drugs.

\section{Conclusions and recommendations}

The use of benralizumab is recommended in patients over 18 years of age with severe uncontrolled eosinophilic asthma (>300 cells $\mu \mathrm{L}^{-1}$ in blood) (level of evidence $1+$, grade of recommendation A - adults) (strong recommendation). The use of benralizumab is not recommended in the paediatric population, as there are no published studies so far. More high-quality randomised studies are required to evaluate the efficacy and safety of benralizumab in patients with severe asthma, mainly in the paediatric population. (We recommend carrying out research, and the use of benralizumab should be based on the results of clinical studies.)

\section{Question 15. What is the efficacy and safety of anti-IL-4 and IL-13 monoclonal antibodies in the} treatment of severe asthma in children and adults?

Justification

IL-13 is a cytokine secreted by T-helper type 2 (Th2) cells, CD4 cells, natural killer T-cells, mast cells, basophil cells and eosinophil cells, among others, and it is a central regulator in IgE secretion, mucus hypersecretion and bronchial muscle contractibility. It shares with IL-4 a multisubunit receptor expressed in several cells involved in the pathophysiology of allergy and asthma. As a result, there is a need to know which benefits are generated by anti-IL-13 drugs and which are generated by anti-IL-4 drugs in the treatment of patients with severe asthma.

Research

Exhaustive research was carried out (192 documents in PubMed, 21 in Embase, 497 in the TRIP database and four in different databases), and seven documents were selected (6 SRs and a post hoc analysis).

\section{Evidence summary}

Lebrikizumab and tralokinumab

Adult patients with uncontrolled asthma, despite the use of medium to high doses of an ICS plus the use of at least $1 \mathrm{~s}$ controller for at least 6 months prior to study entry, were included in the LAVOLTA 1 and LAVOLTA 2 studies. However, in those studies, lebrikizumab did not show a significant reduction in asthma exacerbations versus placebo [41].

ZHANG et al. [42] published a systematic review that compared the efficacy of tralokinumab versus placebo in patients with moderate to severe asthma. The authors included five studies involving 2928 adults. The meta-analysis showed that tralokinumab did not reduce asthma exacerbations or improve asthma-related QoL to a statistically meaningful degree versus placebo. However, tralokinumab did show improvement in $\mathrm{FEV}_{1}$ (MD 0.14 L, 95\% CI: 0.08-0.21, dosage of $300 \mathrm{mg}$ every 2 weeks; MD $0.20 \mathrm{~L}, 95 \%$ CI: $0.01-0.39$, dosage of $600 \mathrm{mg}$ every 4 weeks) and forced vital capacity (MD 0.11,95\% CI: 0.01-0.21). It did not increase the incidence of serious adverse events, but injection site adverse reactions were observed (OR 5.92, 95\% CI: 1.61-21.76). Three phase II controlled clinical trials of patients treated with tralokinumab therapy were taken into consideration. The subjects in those trials were patients with uncontrolled asthma, a history of exacerbations in the previous year, and treatment with medium to high doses of an ICS and an LABA for at least 3 months prior to the beginning of the study. The trials did not show improvement in asthma control, exacerbation rates, $\mathrm{FEV}_{1}, \mathrm{FEF}$ or the QoL. 
Another SR published by Li et al. [43] aimed to evaluate anti-IL-13 monoclonal antibodies. The authors included five studies (involving 3476 patients), two with lebrikizumab and two with tralokinumab. The results of this meta-analysis showed a lower risk of exacerbations in patients receiving anti-IL-13 therapies than in patients receiving placebo (MD 0.19, 95\% CI: -0.27 to -0.11 ). Subgroup analysis showed that patients with high periostin levels $\left(>50 \mathrm{ng} \cdot \mathrm{mL}^{-1}\right)$ had a lower risk of asthma exacerbation (MD 0.30, 95\% CI: -0.41 to -0.19$)$; however, no benefits of anti-IL-13 therapy were shown in patients with low periostin levels (MD 0.06, 95\% CI: -0.18-0.05). Outcomes of $\mathrm{FEV}_{1}$ showed an improvement in patients after receiving anti-IL-13 versus placebo (MD 0.09, 95\% CI: 0.07 to -0.12). There was also an improvement in QoL scores in patients treated with anti-IL-13 versus placebo (MD 0.16, 95\% CI: 0.10-0.21).

\section{Dupilumab}

Dupilumab is a monoclonal antibody directed against the $\alpha$ subunit of the IL- 4 receptor and prevents the signalling of both IL-4 and IL-13, which are two key cytokines in type 2 asthma. Dupilumab is administered subcutaneously. A phase III trial (the Liberty Asthma Quest trial) showed a reduction in asthma exacerbations $(46.9 \%$ - $200 \mathrm{mg}$ and $70.5 \%$ - $300 \mathrm{mg}$ ) with the use of dupilumab compared with placebo. This response was higher in patients with high blood eosinophil levels [41].

In an SR published by Z Z AYED et al. [44], a total of four clinical trials representing 2992 patients were included. A reduction in asthma exacerbation rates was shown in the dupilumab group compared with the placebo group (RR 0.44; 95\% CI: 0.35-0.55). A subanalysis was performed based on blood eosinophil values; the outcome showed a reduction in asthma exacerbations in the patient groups with blood eosinophil counts of $>150$ cells $\cdot \mathrm{mm}^{-3}$. Regarding $\mathrm{FEV}_{1}$ changes, a statistically significant difference was shown in patients receiving dupilumab (MD 0.14 L, 95\% CI: 0.12-0.17). There were no significant differences between the groups in the development of any adverse events (RR 0.99, 95\% CI: 0.95-1.02) or serious adverse events (RR 1.05, 95\% CI: 0.8-1.38). However, there was a higher incidence of discomfort due to injection site reactions in the group receiving dupilumab (RR 1.91, 95\% CI: 1.41-2.59).

In an SR and meta-analysis published by XIONG et al. [45], 5 clinical studies involving 3369 patients were included. The analysis showed significant improvements in lung function in the dupilumab group compared with the placebo group, mainly in $\mathrm{FEV}_{1}$ percentage ( $\mathrm{SMD}=4.29,95 \% \mathrm{CI}$ : $2.78-5.81$ ) and QoL scores $(\mathrm{SMD}=4.39,95 \% \mathrm{CI}: 1.44-7.34)$. There were also significant improvements in asthma symptom control as determined by ACQ-5 score ( $\mathrm{SMD}=-4.95,95 \% \mathrm{CI}:-7.30$ to -2.60$)$ and a reduction in severe exacerbation risks ( $R R=0.73$; 95\% CI: $0.67-0.79)$ in patients receiving dupilumab compared with placebo.

In a recent post hoc analysis of the Liberty Quest study [46], carried out in patients with allergic asthma $(n=1083)$, it was shown that the administration of dupilumab 200/300 mg every 2 weeks versus placebo reduced the asthma exacerbation rate $(36.9 \%$ versus $45.5 \%$; both $\mathrm{p}<0.01)$ and improved the $\mathrm{FEV}_{1}$ at week 12 ( $0.13 \mathrm{~L}$ versus $0.16 \mathrm{~L}$; both $\mathrm{p}<0.001)$.

\section{Indirect comparisons}

EDRIS et al. [41] published a systematic review and network meta-analysis carried out on 30 clinical trials comparing all monoclonal antibodies in patients with severe asthma; however, no significant superiority was observed for one biologic over the others. All of them significantly reduced the risk of exacerbation compared with placebo. Dupilumab and tezepelumab improved lung function in patients with frequent exacerbations.

Another SR and network meta-analysis published by IFTiKHAR et al. [47] included seven trials with benralizumab, two with dupilumab, four with lebrikizumab, seven with mepolizumab, four with reslizumab and two with tralokinumab in subjects with eosinophilic asthma. All drugs were superior to placebo, except for tralokinumab. In terms of the magnitude of effect, dupilumab, followed by reslizumab and benralizumab showed the greatest increase in $\mathrm{FEV}_{1}(0.16 \mathrm{~L}, 95 \% \mathrm{CI}: 0.08-0.24,0.13 \mathrm{~L}, 95 \%$ CI: $0.10-0.17,0.12 \mathrm{~L}, 95 \%$ CI: 0.08-0.17). All drugs except tralokinumab showed reductions in ACQ scores. Mepolizumab was the most effective $(-0.42,95 \%$ CI: -0.55 to -0.29$)$, followed by dupilumab $(-0.31,95 \%$ CI: -0.50 to -0.12$)$, benralizumab $(-0.28,95 \% \mathrm{CI}:-0.38$ to -0.18$)$ and reslizumab $(-0.26,95 \% \mathrm{CI}:-0.39$ to -0.13$)$. In order of the magnitude of effect, dupilumab, followed by mepolizumab, benralizumab and reslizumab, showed the greatest increase in QoL questionnaire scores (0.27, 95\% CI: 0.09-0.45; 0.26, 95\% CI: 0.15, 0.37; 0.26, 95\% CI: $0.10-0.41$, respectively), while tralokinumab showed no significant benefit. Dupilumab and reslizumab decreased asthma exacerbations (RR 0.37, 95\% CI: 0.17-0.80; and RR 0.64, 95\% CI: 0.53-0.78, respectively).

\section{Conclusions and recommendations}

We recommend the use of dupilumab in adult patients with severe allergic and eosinophilic asthma and in adult patients with severe corticosteroid-dependent asthma (level of evidence 1+, grade of recommendation A - adults) (strong recommendation). 
A consistent efficacy of lebrikizumab and tralokinumab therapies in the treatment of patients with severe asthma has not been demonstrated; therefore, we do not suggest their use (level of evidence 1+, grade of recommendation $\mathrm{A}$ - adults) (conditional recommendation).

There are no studies in the paediatric population; therefore, we do not recommend the use of these drugs in paediatric patients (level of evidence 4, recommendation grade D - paediatric patients). (It is recommended to carry out research, and the use of these drugs is dependent on the results of these clinical studies.)

\section{Discussion}

We did not find high-quality evidence to help us recommend the on-demand use of the combination of an ICS plus formoterol treatment in patients with severe asthma. New clinical trials need to be carried out to support this indication. We did not find robust and quality information to recommend the use of tiotropium in patients with severe asthma. Unfortunately, from the detailed analysis of the studies included in the published meta-analyses on tiotropium use in asthma treatment, regarding the inclusion of patients with severe asthma, we found discrepancies in the evaluation of $<52$ weeks and the number of patients included. Our group considered that more clinical studies are needed, complying with internationally accepted definitions, to demonstrate the efficacy and safety of tiotropium therapy in patients with severe asthma. A recommendation was made for the use of omalizumab in patients with severe uncontrolled allergic asthma. The use of anti-IL-5 drugs was recommended for patients with severe uncontrolled eosinophilic asthma. The use of dupilumab was recommended in patients with severe uncontrolled asthma of both allergic and eosinophilic phenotypes and in patients with severe corticosteroid-dependent asthma (table 3). Severe asthma represents a serious health problem, and Latin America is not exempt. Most

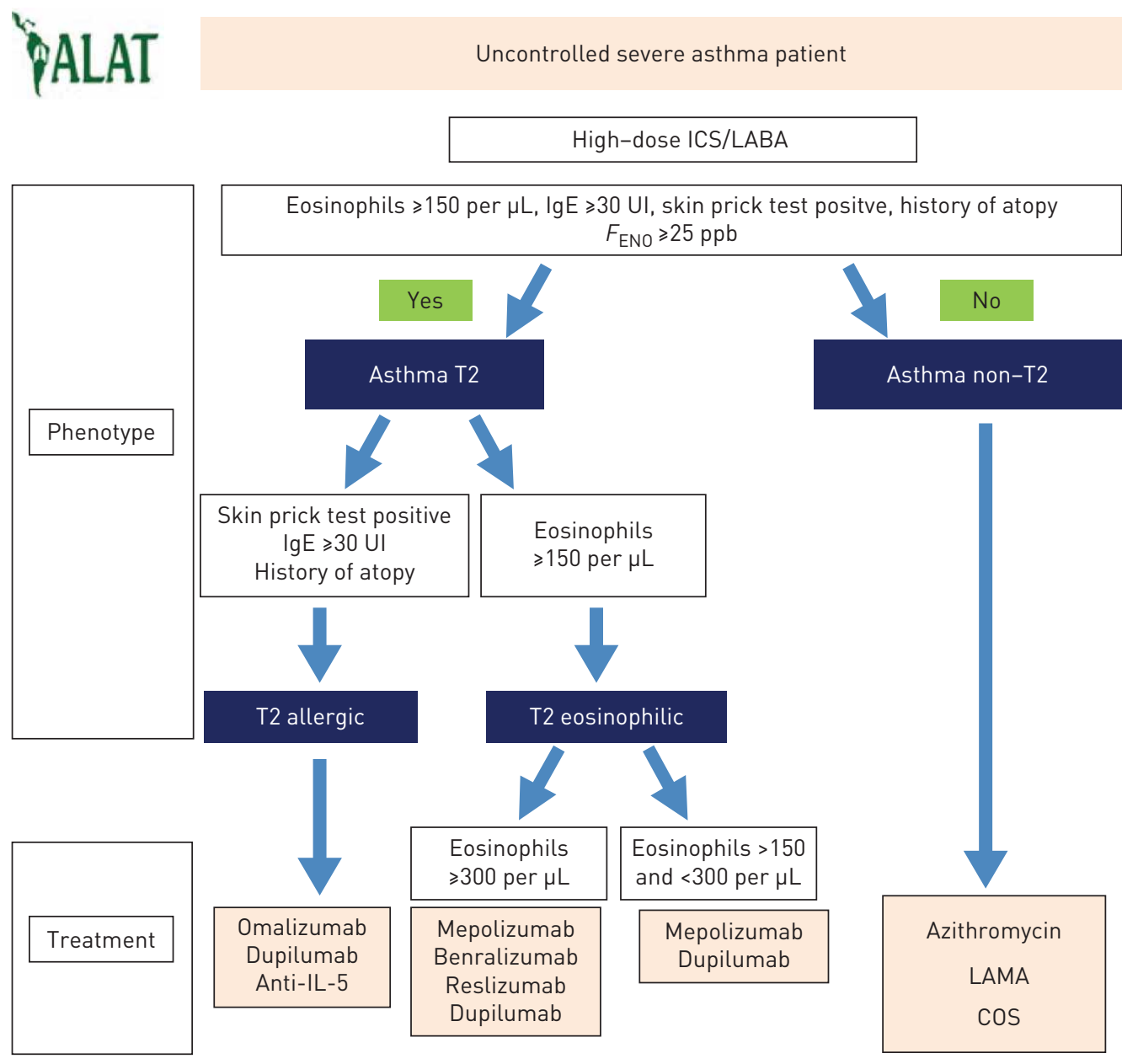

FIGURE 2 Not controlled severe asthma. Treatment algorithm. $F_{\text {ENO: }}$ exhaled nitric oxide fraction; T2: type 2; ICS: inhaled corticosteroid; LABA: long-acting $\beta_{2}$-agonist; IL; interleukin; LAMA: long-acting muscarinic antagonist; COS: oral corticosteroids. 
patients do not have access to an adequate evaluation and treatment of the disease. The profound diversity of the region, for example, in the genetic load, environmental pollution levels, tobacco smoke exposure, geographic and climate differences, and access to poor-quality health systems, has made severe asthma even more serious in Latin America. Throughout many surveys, our asthma department has found that patients as well as healthcare providers have a profound ignorance about severe asthma. A relevant fact is that the acceptance of clinical practice guideline recommendations among professionals treating patients with severe asthma is low.

Our group considered the inclusion of indirect comparisons among biological treatments, as there are no clinical trials with direct comparisons. After analysing all publications with indirect comparisons, we were unable to conclude if one biologic is better than the others. Therefore, new quality information is needed to respond to this concern.

We experienced a great challenge in developing an algorithm to help professionals classify phenotypes and treat patients with severe asthma in Latin America (figure 2). Very few countries in the continent have centres that perform sputum induction procedures and exhaled nitric oxide measurements; therefore, we had to adapt the available information to our reality. Currently, for example, a patient with severe asthma living in Mexico will probably have to travel hundreds of kilometres to access such induced sputum procedures. Another example is Argentina, where, for instance, the measurement of exhaled nitric oxide has not yet been approved. All these disadvantages were considered when we developed the algorithm, with the understanding that there is a possible overlap in patients. Last, based on the problems already mentioned, we have considered not only the recommendations mentioned above but also drug access and availability in each country.

In summary, the questions analysed and presented in this document allowed our ALAT Asthma Department group to make recommendations on the treatment of severe asthma adapted to real-life situations in Latin America. We expect that these recommendations will help professionals improve their knowledge and will assist in decision-making by helping the health system manage resources more accurately and conveniently. It will be important for our colleagues in the region to individualise the treatment approach for each patient, and for each variable that makes the management of this disease so complex, with even more complex clinical settings and resources.

Conflict of interest: G. García reports research grants from Novartis, GSK, Boehringer Ingelheim, AstraZeneca and Sanofi, and acting as a consultant/advisor/speaker for GSK, Astra Zeneca, Novartis and Sanofi. M. Bergna reports research grants from Novartis, GSK, Boehringer Ingelheim, Roche, AstraZeneca and Sanofi, and acting as a consultant/ advisor/speaker for GSK, AstraZeneca and Novartis. J.C. Vazquez reports acting as a consultant/advisor/speaker for GSK, AstraZeneca, Novartis and Boehringer Ingelheim. M.C. Cano Salas reports acting as a consultant/advisor/speaker for GSK, AstraZeneca, Novartis and Boehringer Ingelheim. J.L. Miguel reports acting as a consultant/advisor/speaker for GSK, AstraZeneca, Novartis and Boehringer Ingelheim. C. Celis Preciado reports research grants from Novartis and AstraZeneca. A. Acuña Izcaray reports acting as a consultant/advisor/speaker for GSK, AstraZeneca, Novartis and Boehringer Ingelheim. M. Barros Monge reports research grants from Novartis, GSK, Boehringer Ingelheim and AstraZeneca, and acting as a consultant/advisor/speaker for GSK, AstraZeneca and Novartis. N. Garcia Batista reports acting as a consultant/advisor/speaker for AstraZeneca and Novartis. I. Zabert has nothing to disclose. J.L. Mayorga has nothing to disclose. R. Casanova Mendoza has nothing to disclose. M. Gutierrez has nothing to disclose. M.F. Montero Arias reports research grants from Novartis. L. Utrecho Perez has nothing to disclose. M. Antunez reports acting as a consultant/advisor/speaker for GSK, AstraZeneca, Novartis and Boehringer Ingelheim. V. Williams Derby has nothing to disclose. A. Villatoro Azméquita has nothing to disclose. L. Motiño has nothing to disclose. M.A. De Oliveira has nothing to disclose. D. Rey Sanchez has nothing to disclose. M. Arroyo has nothing to disclose. M. Rodriguez has nothing to disclose.

\section{References}

1 Bousquet J, Mantzouranis E, Cruz AA, et al. Uniform definition of asthma severity, control, and exacerbations: document presented for the World Health Organization Consultation on Severe Asthma. J Allergy Clin Immunol 2010; 126: 926-938.

2 NHLBI. U.S. Department of Health and Human Services, National Institutes of Health [Internet]. Expert Panel Report 3: Guidelines for the Diagnosis and Management of Asthma (EPR-3). Bethesda, National Heart, Lung, and Blood Institute (US), 2007. https://www.ncbi.nlm.nih.gov/books/NBK7232/

3 Chung KF, Wenzel SE, Brozek JL, et al. International ERS/ATS Guidelines on Definition, Evaluation, and Treatment of Severe Asthma. Eur Respir J 2014; 43: 343-373.

4 Ocampo J, Gaviria R, Sánchez J. Prevalence of asthma in Latin America. Critical look at ISAAC and other studies. Rev Alerg Mex 2017; 64: 188-197.

5 Forno E, Gogna M, Cepeda A, et al. Asthma in Latin America. Thorax 2015; 70: 898-905.

6 Neffen $\mathrm{H}$, Chahuàn $\mathrm{M}$, Hernández $\mathrm{DD}$, et al. Key factors associated with uncontrolled asthma - the Asthma Control in Latin America Study. J Asthma 2020; 57: 113-122.

7 Solé D, Aranda CS, Wandalsen GF. Asthma: epidemiology of disease control in Latin America - short review. Asthma Res Pract 2017; 3: 4-9. 
8 Nathan RA, Thompson PJ, Price D, et al. Taking Aim at Asthma Around the World: Global Results of the Asthma Insight and Management Survey in the Asia-Pacific Region, Latin America, Europe, Canada, and the United States. J Allergy Clin Immunol Pract 2015; 3: 734-742.

9 Bate L, Hutchinson A, Underhill J, et al. How clinical decisions are made. Br J Clin Pharmacol 2012; 74: 614-620.

10 Brouwers MC, Kerkvliet K, Spithof K. The AGREE reporting checklist: a tool to improve reporting of clinical practice guidelines. BMJ 2016; 352: i1152.

11 Shea BJ, Reeves BC, Wells G, et al. AMSTAR 2: a critical appraisal tool for systematic reviews that include randomised or non-randomised studies of healthcare interventions, or both. BMJ 2017; 358: j4008.

12 Tao H, Yang L, Ping A, et al. Interpretation of AMSTAR 2: A critical appraisal tool for systematic reviews that include randomized or non-randomized studies of healthcare interventions. Chin Evid-Based Med 2018; 18 101-108.

13 von Elm E, Altman DG, Egger M, et al. Strengthening the reporting of observational studies in epidemiology (STROBE) statement: Guidelines for reporting observational studies. Int J Surg 2014; 12: 1495-1499.

14 Bossuyt PM, Reitsma JB, Bruns DE, et al. STARD 2015: an updated list of essential items for reporting diagnostic accuracy studies. Clin Chem 2015; 61: 1446-1452.

15 Cates CJ, Lasserson TJ. Combination formoterol and inhaled steroid versus beta 2-agonist as relief medication for chronic asthma in adults and children. Cochrane Database Syst Rev 2009; 1: CD007085.

16 Cates CJ, Karner C. Combination formoterol and budesonide as maintenance and reliever therapy versus current best practice (including inhaled steroid maintenance), for chronic asthma in adults and children. Cochrane Database Syst Rev 2013; 30: CD007313.

17 Sobieraj DM, Weeda ER, Nguyen E, et al. Association of inhaled corticosteroids and long-acting $\beta$-agonists as controller and quick relief therapy with exacerbations and symptom control in persistent asthma: a systematic review and meta-analysis. JAMA 2018; 319: 1485-1496.

18 Rodrigo GJ, Castro-Rodríguez JA. What is the role of tiotropium in asthma?: a systematic review with meta-analysis. Chest 2015; 147: 388-396.

19 Kew K, Dahri K. Long-acting muscarinic antagonists (LAMA) added to combination long-acting beta2-agonists and inhaled corticosteroids (LABA/ICS) versus LABA/ICS for adults with asthma. Cochrane Database Syst Rev 2016; 1: CD011721.

20 Rodrigo GJ, Castro-Rodríguez JA. Tiotropium for the treatment of adolescents with moderate to severe symptomatic asthma: a systematic review with meta-analysis. Ann Allergy Asthma Immunol 2015; 115: 211-216.

21 Szefler SJ, Murphy K, Harper T, et al. A phase III randomized controlled trial of tiotropium add-on therapy in children with severe symptomatic asthma. J Allergy Clin Immunol 2017; 140: 1277-1287.

22 Normansell R, Walker S, Sj M, et al. Omalizumab for asthma in adults and children. Cochrane Database Syst Rev 2014; 1: CD003559.

23 Norman G, Faria R, Paton F, et al. Omalizumab for the Treatment Severe persistent Allergic asthma: a Systematic Review and Economic Evaluation. Heal Technol Assess 2013; 17: 1-342.

24 Fitzgerald JM, Lemiere C, Lougheed MD, et al. Recognition and management of severe asthma: a Canadian Thoracic Society position statement. Can J Respir Crit Care Sleep Med 2017; 1: 199-221.

25 Global Initiative for Asthma. Global Strategy for Asthma Management and Prevention. 2019. www.ginasthma.org/ 2019-gina-report-global-strategy-for-asthma-management-and-prevention Date last accessed: October 28, 2019.

26 Nachef Z, Krishnan A, Mashtare T, et al. Omalizumab versus Mepolizumab as add-on therapy in asthma patients not well controlled on at least an inhaled corticosteroid: a network meta-analysis. J Asthma 2018; 55: 89-100

27 Liu Y, Zhang S, Li DW, et al. Efficacy of anti-interleukin-5 therapy with mepolizumab in patients with asthma: a meta-analysis of randomized placebo-controlled trials. PLoS One 2013; 8: e59872.

28 Yancey SW, Ortega HG, Keene ON, et al. Meta-analysis of asthma-related hospitalization in mepolizumab studies of severe eosinophilic asthma. J Allergy Clin Immunol 2017; 139: 1167-1175.e2.

29 Powell C, Sj M, Dwan K, et al. Mepolizumab versus placebo for asthma [Review]. Cochrane Database Syst Rev 2015; 7: CD010834.

30 Farne HA, Wilson A, Powell C, et al. Anti-IL5 therapies for asthma. Cochrane Database Syst Rev 2017; 9 : CD010834

31 Li J, Wang F, Lin C, et al. The efficacy and safety of reslizumab for inadequately controlled asthma with elevated blood eosinophil counts: a systematic review and meta-analysis. J Asthma 2017; 54: 300-307.

32 Henriksen DP, Bodtger U, Sidenius K, et al. Efficacy, adverse events, and inter-drug comparison of mepolizumab and reslizumab anti- IL-5 treatments of severe asthma - a systematic review and meta-analysis. Eur Clin Respir J 2018; 5: 1536097.

33 Casale TB, Pacou M, Mesana L, et al. Reslizumab compared with benralizumab in patients with eosinophilic asthma: a systematic literature review and network meta-analysis. J Allergy Clin Immunol Pract 2019; 7: 122-130.

34 He L, Zhang L, Jiang L, et al. Efficacy and safety of anti-interleukin-5 therapy in patients with asthma: a pairwise and Bayesian network meta-analysis. Int Immunopharmacol 2018; 64: 223-231.

35 National Institute for Health and Care Excellence. Technology appraisal guidance (TA565). Benralizumab for treating severe eosinophilic asthma (2019). www.nice.org.uk/guidance/ta565/

36 Tian B, Zhang G, Lou J, et al. Efficacy and safety of benralizumab for eosinophilic asthma: A systematic review and meta-analysis of randomized controlled trials and meta-analysis of randomized controlled trials. J Asthma 2018; 55: 956-965.

37 Liu T, Wang F, Wang G, et al. Efficacy and safety of benralizumab in patients with eosinophilic asthma: a meta-analysis of randomized placebo-controlled trials. Front Med 2018; 12: 340-349.

38 Liu W, Zhou W. Adverse events of benralizumab in moderate to severe eosinophilic asthma. Medicine (Baltimore) 2019; 98: e15868.

39 Cabon Y, Molinari N, Marin G, et al. Comparison of anti-interleukin-5 therapies in patients with severe asthma: global and indirect meta-analyses of randomized placebo-controlled trials. Clin Exp Allergy 2017; 47: 129-138.

40 Bourdin A, Husereau D, Molinari N, et al. Matching adjusted indirect comparison of benralizumab versus interleukin5 inhibitors for the treatment of severe asthma: a systematic review. Eur Respir J 2018; 52: 1801393.

41 Edris A, De Feyter S, Maes T, et al. Monoclonal antibodies in type 2 asthma: a systematic review and network meta-analysis. Respir Res 2019; 20: 179. 
42 Zhang Y, Cheng J, Li Y, et al. The safety and efficacy of anti-IL-13 treatment with tralokinumab (CAT-354) in moderate to severe asthma: a systematic review and meta-analysis. J Allergy Clin Immunol Pract 2019; 7 : 2661-2671.

43 Li H, Wang $\mathrm{K}$, Huang $\mathrm{H}$, et al. A meta-analysis of anti-interleukin-13 monoclonal antibodies for uncontrolled asthma. PLoS One 2019; 14: 1-13.

44 Zayed Y, Kheiri B, Banifadel M, et al. Dupilumab safety and efficacy in uncontrolled asthma: a systematic review and meta-analysis of randomized clinical trials. J Asthma 2019; 56: 1110-1119.

45 Xiong XF, Zhu M, Wu HX, et al. Efficacy and safety of dupilumab for the treatment of uncontrolled asthma: a meta-analysis of randomized clinical trials. Respir Res 2019; 20: 108.

46 Corren J, Castro M, O’Riordan T, et al. Dupilumab efficacy in patients with uncontrolled, moderate-to-severe allergic asthma. J Allergy Clin Immunol Pract 2020; 8: 516-526.

47 Iftikhar IH, Schimmel M, Bender W, et al. Comparative efficacy of anti IL-4, IL-5 and IL-13 drugs for treatment of eosinophilic asthma: a network meta-analysis. Lung 2018; 196: 517-530. 